\title{
Comparative Effectiveness of Ezetimibe in Improving Lipid Profile in Non-Alcoholic Fatty Liver Disease Patients: Statins Still Rule
}

\author{
Mohammad Ebrahim Ghamar-Chehreh, Mohsen Amini, Hossein Khedmat (Corresponding author) \\ Fatemeh Daraei, Reza Mohtashami \& Ashraf Karbasi \\ The Internist Research Center, Baqiyatallah Research Center for Gastroenterology and Liver Diseases \\ Baqiyatallah University of Medical Sciences, Tehran, Iran \\ E-mail: khedmat.h@gmail.com
}

Saeed Taheri

Dr. Taheri Medical Research Group, Tehran, Iran

Received: December 5, 2011

Accepted: December 16, $2011 \quad$ Published: April 1, 2012

doi:10.5539/ijb.v4n2p184

URL: http://dx.doi.org/10.5539/ijb.v4n2p184

\begin{abstract}
Introduction: Dyslipidemias are well-documented disorders in nonalcoholic fatty liver disease (NAFLD) patients; and have been implicated as an etiology and prognosis factor for the disease, as well. In the current study, we aimed to evaluate the impact of various antihyperlipidemic regimens on the serum cholesterol levels.

Method and material: A longitudinally study was conducted and 98 NAFLD patients were consecutively entered into analysis. Five types of antihyperlipidemic regimens were used: Ezetimibe alone (10mg/day; 3 patients); Ezetimibe (10mg/day) plus gemfibrozil (600mg/day) (28 patients); Gemfibrozil alone (600mg/day; 26 patients); Atorvastatin (20mg/day; 15 patients); and Simvastatin (20mg/day; 26 patients).

Results: patients were followed for $138 \pm 83$ days after drugs administered. Serum total cholesterol levels experiences highest fall by atorvastatin therapy $(\mathrm{p}=0.006)$. Serum LDL levels also decreased most significantly through antihyperlipidemic therapy with atorvastatin or simvastatin $(p<0.001)$. There was no difference between the study drug regimens modifying serum HDL, triglyceride, AST and ALT levels ( $p>0.1$ for all).

Conclusion: Ezetimibe therapy alone cannot improve hyperlipidemias in NAFLD patients, while statins, especially atorvastatin is highly potent for this purpose; and is recommended for hyperlipidemic NAFLD patients. For NAFLD patients who have unfavorable reactions to statins and are subjected to use ezetimibe, we recommend its co-administration with gemfibrozil. Future studies with randomized and blind approaches are needed.
\end{abstract}

Keywords: Non-alcoholic fatty liver disease, Hyperlipidemia, Treatment, Drug

\section{Introduction}

Non alcoholic fatty liver disease (NAFLD) ranges from pure lipid accumulation in hepatocytes to nonalcoholic steatohepatitis (NASH), a condition that can be associated with necroinflammation and fibrosis; and to end stage liver disease and hepatocellular carcinoma (Khedmat and Taheri, 2011). There are several endocrine disorders which have been associated with NAFLD from which glycemic and lipidemic disorders are the major ones. Insulin resistance (IR) is considered to play central role in the pathogenesis of NAFLD (Angelico et al., 2006; Patel et al., 2004). Moreover, dyslipidemias are well-documented disorders in nonalcoholic fatty liver disease (NAFLD) patients; and have been implicated as etiologic and prognostic factors for the disease, as well.

Ezetimibe is expected to inhibit the absorption of both dietary and biliary cholesterol in the small intestine (Davies et al., 2005), and has been approved to decrease serum cholesterol values in human beings. Moreover, ezetimibe has been shown to ameliorate liver steatosis and cholesterol gallstone formation in animal models (Altmann et al., 2004; Kosoglou et al., 2005). Current evidence suggests that ezetimibe has some beneficial effects in the treatment of NAFLD. However, most of these data have been derived from animal models, and 
there is data scarcity in the literature on the potential effects of ezetimibe or drug regimens including this agent on NAFLD biochemical measures.

Current literature suggests that ezetimibe improves insulin resistance, hepatic insulin sensitivity, and fat accumulation; as main factors occurring early in the pathogenesis of NAFLD (Zheng et al., 2008; Expert Panel, 2001); however, most of the existing data are from studies indirectly issue it; from which only a limited number are on humans. So, to address the issue of the magnitude of the effects of ezetimibe therapy in humans with NAFLD, and to compare changes in disease parameters with other antihyperlipidemic agents, we conducted a longitudinal study in which hyperlipidemic NAFLD patients undergone pharmacologic therapy with different drug regimens; and after the follow up, we compared changes in the disease measures, including serum lipid profile and aminotransferase levels to find the magnitude of the impact of ezetimibe therapy on NAFLD.

\section{Methods and Material}

A longitudinally study was conducted involving the outpatient Gastroenterology Clinic from Baqiyatallah University of Medical Sciences, Tehran, Iran. 188 consecutive individuals who attended to our clinic, and had hyper cholesterolemia and finally were diagnosed as having NAFLD entered into the study, but only 98 of them came at the time for follow up laboratory tests and were entered into analysis. The study participants comprised a full range of socioeconomic levels.

\subsection{Ethics, drug regimens \& study groups}

The study was approved by the local Ethics Committee of the Baqiyatallah University of Medical Sciences and written informed consent for participation was obtained from all the participants. Due to the proved effect of statins and gemfibrozil in reducing serum lipid levels, ethics committee only admitted to administer ezetimibe alone, to NAFLD patients who had a previous history for unfavorable reaction to statins. So five different drug regimens were used: (1) Ezetimibe alone (10mg/day; 3 patients); (2) Ezetimibe (10mg/day) plus gemfibrozil (600mg/day) (28 patients); (3) Gemfibrozil alone (600mg/day; 26 patients); (4) Atorvastatin (20mg/day; 15 patients); and (5) Simvastatin (20mg/day; 26 patients).

\subsection{Anthropometrical measures}

Anthropometrical measurements (including height, weight, blood pressure, waist/hip diameter, etc) were collected by well-trained nurses while patients wore light clothing and no shoes. Waist circumference was measured - with the subject standing and wearing only underwear, at the level midway between the lower rib margin and the iliac crest. Body mass index (BMI) was calculated as weight $(\mathrm{Kg})$ divided by height $(\mathrm{m})$ squared $\left(\mathrm{kg} / \mathrm{m}^{2}\right)$.

\subsection{Blood samples}

Blood samples were taken in $0.1 \%$ EDTA in morning and after 12 hours fast. Blood tubes were immediately stored at $41 \mathrm{C}$ and shielded from light. Blood tubes were centrifuged within $6 \mathrm{~h}$ at $2500 \mathrm{rpm}$ for $20 \mathrm{~min}$ to separate plasma. Serum concentrations of total cholesterol, fasting triglycerides, glucose, LDL- and HDL-cholesterol (LDL-C, HDL-C plus 2nd generation, direct quantification) were determined by using enzymatic kits from Roche Diagnostics with an autoanalyzer. Cholesterol measurements were standardized according to the program specified by the Centers for Disease Control and the National Heart, Lung and Blood Institute.

Homeostatic model assessment (HOMA) was calculated with the following formula: insulin (mIU/L)* glucose (in $\mathrm{mmol} / \mathrm{L}$ ) $/ 22.5$. ALT levels were considered pathologically high when it was over 30 for men and over 19 for women.

\subsection{Metabolic syndrome}

According to the third report of the Adult Treatment Panel (ATP III), NAFLD patients with three or more of the following criteria were defined as having the metabolic syndrome (Park et al., 2011): (1) abdominal girth: waist girth over $102 \mathrm{~cm}$ in men or $88 \mathrm{~cm}$ in women; (2) hypertriglyceridemia: serum triglycerides over $150 \mathrm{mg} / \mathrm{dl}$; (3) low high-density lipoprotein cholesterol: serum HDL cholesterol below $40 \mathrm{mg} / \mathrm{dl}$ in men or $50 \mathrm{mg} / \mathrm{dl}$ in women; (4) high blood pressure: blood pressure over 130/85 mmHg; (5) high fasting glucose: blood glucose levels exceeding $100 \mathrm{mg} / \mathrm{dl}$. Subjects using antihypertensive medication were considered to meet the criteria for high blood pressure.

\subsection{Data analysis}

For descriptive data, one way ANOVA was used; and multiple comparisons were performed using Bonferoni's test. Chi square test was used for categorical variables. Likelihood estimates (Odds ratio) and $95 \%$ confidence 
intervals (CI) were obtained by use of logistic regression. A value of $p<0.05$ on the two-tail test was considered statistically significant. Statistical analyses were performed using SPSS-17.0 (SPSS Corp.; IL; Chicago; USA) for Windows.

\section{Results}

There were $48(49 \%)$ males and $50(51 \%)$ females. Mean \pm SD age of the study population was $47.2 \pm 9$ years. Self-rated socioeconomic levels were $91(93 \%)$ intermediate and $7(7 \%)$ low. Educational levels of the study participants were $53(54 \%)$ high school diploma, 34 (35\%) under diploma, 1 (1\%) illiterate, and $10(10 \%)$ bachelor of science or more.

Patients under different antihypercholesterolemic regimens experienced comparable change in their liver enzymes including AST $(p=0.898)$ and ALT $(p=0.765)$ values. Moreover, no priority was found for any of the regimens regarding treatment of insulin resistance $(\mathrm{P}=0.867)$ and metabolic syndrome $(\mathrm{P}=0.421)$. Moreover, none of the regimens had significantly higher effect on improving serum glucose and HDL levels and blood pressure ( $\mathrm{p}>0.05$ for both).

Table 1 summarizes changes in the study variables using different drug regimens. As can be seen, changes in the lipid profiles of our NAFLD patients were significantly different regarding their antihyperlipidemic regimens. Serum total cholesterol levels experiences highest fall by atorvastatin therapy $(\mathrm{p}=0.006$; figure 1$)$. Serum LDL levels also decreased most significantly through antihyperlipidemic therapy with atorvastatin or simvastatin $(\mathrm{p}<0.001$; figure 2). Gemfibrozil alone had no superior lowering effect on serum cholesterol and LDL values compared to others; as well, ezetimibe had no significant role in decreasing any of the serum lipid levels, and there was slight elevation in them after using ezetimibe alone. But putting together, ezetimibe and gemfibrozil had higher impact on lowering serum cholesterol and LDL values. Except for ezetimibe alone, all other drug regimens reduced serum triglyceride levels, but there was no significant difference between $(\mathrm{p}=0.36$; figure 3 ).

\section{Discussion}

In this study, we found that ezetimibe therapy in NAFLD patients with hypercholesterolemia, if is not accompanied by any other antihyperlipidemic drugs, has no beneficial effect on total cholesterol and LDL values. Nor it has any favorable effect on liver enzyme levels including AST and ALT. Moreover, ezetimibe alone was found not effective in reducing rates of insulin resistance or metabolic syndrome or their components. To our knowledge, this study is one of the premier studies in the literature that investigates the effects of ezetimibe therapy on the disease components of human NAFLD patients, comparing its effects with other conventional antihyperlipidemic treatments. Previous studies mostly target other patient populations or animals; although there is some limited data on humans too. Park et al. (Park et al., 2011), in their study have showed beneficial effects for ezetimibe therapy on metabolic, biochemical, and histological abnormalities of NAFLD. However, their study was not a controlled one; and authors only compared data of their patients before and after the treatment. Based on these results, some authors have suggested ezetimibe as an effective treatment of NAFLD (Ahmed et al., 2010). So, addressing this issue, our study found no beneficial effects for ezetimibe therapy on NAFLD parameters than that achieved by statins. Our data is confirmatory to presumptions suggesting statins as the best treatment for NAFLD patients (Athyros et al., 2011; Musso et al., 2011).

Despite comprehensive search we did only find limited studies addressing antihyperlipidemic effects of ezetimibe in NAFLD patients. In a previous study on diabetic patients without NAFLD, Goldberg et al. (Goldberg, 2006), have compared lipid lowering effects of ezetimibe and simvastatin co-administration versus atorvastatin alone. They found that co-administration of ezetimibe and simvastatin has superior impact on LDL and triglyceride reduction compared to atorvastatin alone. Due to the documented effects of statins on lipid profile, however in the current study, the effects of co-administration of ezetimibe and gemfibrozil were compared to that of statins. We found an elevated activity for ezetimibe treatment when it is co-administered with gemfibrozil; although even after combination therapy, its antihyperlipidemic effect did not surpass statins'. A previous study also suggested that ezetimibe co-administration with orlistat can be effective on NAFLD biochemical markers in obese and hyperlipidemic patients with no documented NAFLD diagnosis (Filipatos, 2011). Furthermore, ezetimibe plus statins has also been confirmed as a highly effective treatment protocol for hypercholesterolemias in different patient populations (Assmann et al., 2008; Bennett et al., 2004; Simons et al., 2004; Goldberg et al., 2004).

There is a previous study that has shown beneficial effects for ezetimibe in reducing serum liver enzymes including ALT and AST in NASH patients (Yoneda et al., 2010). Another study on human subjects with NAFLD also showed ezetimibe therapy as an effective method in lowering serum ALT levels in non-obese NAFLD patients (Enjoji et al., 2010). In non-NAFLD context, Chan et al. (Chan et al., 2010), also showed that 
ezetimibe therapy in obese patients committing weight loss can reduce serum LDL levels. However, despite an overall reduction in liver enzymes; compared to other regimens, we did not find any superiority for favorable changes in serum cholesterol or AST/ALT levels after ezetimibe administration in the current study. Nevertheless, we found that atorvastatin was more effective than ezetimibe in reducing either serum total cholesterol or LDL values. One explanation for this observation is that our study did compare effects of ezetimibe therapy with other regimens; and not just within the same group, before and after the treatment. Moreover, our study population has not experienced weight loss; and ezetimibe might only be effective in patients with normal body weight or who lose their weight. On the other hand, there might be some types of selection bias in our study population, while patients in the ezetimibe group were patients who had a history of unfavorable reaction to statins. To our knowledge, there is no data in the literature regarding a potential different disease behavior or response to treatment for NAFLD patients who have reaction to statins.

This study has some limitations. First of all, our study participants were not randomized to subgroups. Moreover, our study was an open labeled study which reduces the strength of our results. The limited number of patients participated in the ezetimibe group - mainly due to the selection criteria and consent giving process - was another confounding factor in this study. So, one may claim that the minimal founded effects of ezetimibe on improving lipid profile in NAFLD patients might be described by its small sample size. However, we should note that even within the group; ezetimibe alone did not reduce serum cholesterol levels after the treatment course in any of the patients. On the other hand, there was another group consisted of 28 patients for whom ezetimibe was co-administered with gemfibrozil. The lipid lowering power of the latter group also, despite ezetimibe employment, did not reach to statins. A point of power in our study can also be the relatively larger population compared to the previous human studies on the same topic; as well as employing 5 drug regimens instead of only two.

In conclusion, we found that ezetimibe therapy alone cannot improve hyperlipidemias in NAFLD patients, while statins, especially atorvastatin is highly potent for this purpose; and is recommended for hyperlipidemic NAFLD patients. For NAFLD patients who have unfavorable reactions to statins and are subjected to use ezetimibe, we recommend its co-administration with gemfibrozil. Future double blind studies with larger study population and randomization of the study population seem necessary for confirming our results.

\section{References}

Abdeen M. B., Chowdhury N. A., Hayden M. R., \& Ibdah J. A. (2006). Nonalcoholic steatohepatitis and the cardiometabolic syndrome. J Cardiometab Syndr, 1, 36-40. http://dx.doi.org/10.1111/j.0197-3118.2006.05523.x

Ahmed M. H., \& Byrne C. D. (2010). Ezetimibe as a potential treatment for non-alcoholic fatty liver disease: is the intestine a modulator of hepatic insulin sensitivity and hepatic fat accumulation? Drug Discov Today, 15(15-16), 590-5. http://dx.doi.org/10.1016/j.drudis.2010.06.007

Altmann S. W., Davis H. R. Jr., Zhu L. J., Yao X., Hoos L. M., Tetzloff G, et al. (2004). Niemann-Pick C1 Like 1 protein is critical for intestinal cholesterol absorption. Science, 303, 1201-4. http://dx.doi.org/10.1126/science.1093131

Angelico F., Del Ben M., Conti R., et al. (2005). Insulin resistance, the metabolic syndrome, and nonalcoholic fatty liver disease. J Clin Endocrinol Metab, 90, 1578-1582. http://dx.doi.org/10.1210/jc.2004-1024

Assmann G., Kannenberg F., Ramey D. R., Musliner T. A., Gutkin S. W., \& Veltri E. P. (2008). Effects of ezetimibe, simvastatin, atorvastatin, and ezetimibe-statin therapies on non-cholesterol sterols in patients with primary hypercholesterolemia. Curr Med Res Opin, 24(1), 249-59. http://dx.doi.org/10.1185/030079908X253663

Athyros V. G., Tziomalos K., Daskalopoulos G. N., Karagiannis A., \& Mikhailidis D. P. (2011). Statin-based treatment for cardiovascular risk and non-alcoholic fatty liver disease. Killing two birds with one stone? Ann Med, 43(3), 167-71. http://dx.doi.org/10.3109/07853890.2011.561363

Bennett S., Sager P., Lipka L., Melani L., Suresh R., \& Veltri E. (2004). Ezetimibe Study Group. Consistency in efficacy and safety of ezetimibe coadministered with statins for treatment of hypercholesterolemia in women and men. J Womens Health (Larchmt), 13(10), 1101-7. http://dx.doi.org/10.1089/jwh.2004.13.1101

Chan D.C., Watts G. F., Gan S. K., Ooi E. M., \& Barrett P. H. (2010). Effect of ezetimibe on hepatic fat, inflammatory markers, and apolipoprotein B-100 kinetics in insulin-resistant obese subjects on a weight loss diet. Diabetes Care, 33(5), 1134-9. http://dx.doi.org/10.2337/dc09-1765

Davies J. P., Scott C., Oishi K., Liapis A., \& Ioannou Y. A. (2005). Inactivation of NPC1L1 causes multiple lipid transport defects and protects against diet-induced hypercholesterolemia. J Biol Chem, 280, 12710-20. http://dx.doi.org/10.1074/jbc.M409110200 
Enjoji M., Machida K., Kohjima M., Kato M., Kotoh K., Matsunaga K., Nakashima M., \& Nakamuta M. (2010). NPC1L1 inhibitor ezetimibe is a reliable therapeutic agent for non-obese patients with nonalcoholic fatty liver disease. Lipids Health Dis, 9, 29. http://dx.doi.org/10.1186/1476-511X-9-29

Expert Panel on Detection, Evaluation, and Treatment of High Blood Cholesterol in Adults. (2001). Executive Summary of the Third Report of The National Cholesterol Education Program (NCEP) Expert Panel on Detection, Evaluation, And Treatment of High Blood Cholesterol In Adults (Adult Treatment Panel III). JAMA, 285(19), 2486-97. http://dx.doi.org/10.1001/jama.285.19.2486

Filippatos T. D., \& Elisaf M. S. (2011). Role of ezetimibe in non-alcoholic fatty liver disease. World J Hepatol, 3(10), 265-7. http://dx.doi.org/10.4254/wjh.v3.i10.265

Goldberg A. C., Sapre A., Liu J., Capece R., \& Mitchel Y. B. (2004). Ezetimibe Study Group. Efficacy and safety of ezetimibe coadministered with simvastatin in patients with primary hypercholesterolemia: a randomized, double-blind, placebo-controlled trial. Mayo Clin Proc, 79(5), 620-9. http://dx.doi.org/10.4065/79.5.620

Goldberg R. B., Guyton J. R., Mazzone T., et al. (2006). Ezetimibe/simvastatin vs atorvastatin in patients with type 2 diabetes mellitus and hypercholesterolemia: the VYTAL study. Mayo Clin Proc, 81(12), $1579-88$. http://dx.doi.org/10.4065/81.12.1579

Khedmat H., Taheri S. (2011). Non-alcoholic steatohepatitis: An update in pathophysiology, diagnosis and therapy. Hepat Mon, 11(2), 74-85.

Kosoglou T., Statkevich P., Johnson-Levonas A. O., Paolini J. F., Bergman A. J., \& Alton K. B. (2005). Ezetimibe: a review of its metabolism, pharmacokinetics and drug interactions. Clin Pharmacokinet, 44(5), 467-94. http://dx.doi.org/10.2165/00003088-200544050-00002

Musso G., Cassader M., \& Gambino R. (2011). Cholesterol-lowering therapy for the treatment of nonalcoholic fatty liver disease: an update. Curr Opin Lipidol, 22(6), $489-96$. http://dx.doi.org/10.1097/MOL.0b013e32834c37ee

Park H., Shima T., Yamaguchi K., et al. (2011). Efficacy of long-term ezetimibe therapy in patients with nonalcoholic fatty liver disease. J Gastroenterol, 46(1), 101-7. http://dx.doi.org/10.1007/s00535-010-0291-8

Patel S. B. (2004). Ezetimibe: a novel cholesterol-lowering agent that highlights novel physiologic pathways. Curr Cardiol Rep, 6, 439-42. http://dx.doi.org/10.1007/s11886-004-0052-5

Simons L., Tonkon M., Masana L., Maccubbin D., Shah A., Lee M., \& Gumbiner B. (2004). Effects of ezetimibe added to on-going statin therapy on the lipid profile of hypercholesterolemic patients with diabetes mellitus or metabolic syndrome. Curr Med Res Opin, 20(9), 1437-45. http://dx.doi.org/10.1185/030079904X2321

Yoneda M., Fujita K., Nozaki Y., et al. (2010). Efficacy of ezetimibe for the treatment of non-alcoholic steatohepatitis: An open-label, pilot study. Hepatol Res, 40(6), 613-21. http://dx.doi.org/10.1111/j.1872-034X.2010.00644.x

Zheng S., Hoos L., Cook J., Tetzloff G., Davis H. Jr, van Heek M., \& Hwa J. J. (2008). Ezetimibe improves high fat and cholesterol diet-induced non-alcoholic fatty liver disease in mice. Eur $J$ Pharmacol, 584(1), 118. http://dx.doi.org/10.1016/j.ejphar.2008.01.045

Table 1. Changes in the biological parameters of the study participants

\begin{tabular}{|c|c|c|c|c|c|c|}
\hline & $\begin{array}{l}\text { Regimen } \\
1\end{array}$ & $\begin{array}{l}\text { Regimen } \\
2\end{array}$ & $\begin{array}{l}\text { Regimen } \\
3\end{array}$ & $\begin{array}{l}\text { Regimen } \\
4\end{array}$ & $\begin{array}{l}\text { Regimen } \\
5\end{array}$ & Sig. \\
\hline \multicolumn{7}{|l|}{ Changes in: } \\
\hline Total cholesterol $(\mathrm{mg} / \mathrm{dL})$ & $39 \pm 34$ & $-28 \pm 45$ & $-4 \pm 36$ & $-46 \pm 54$ & $-34 \pm 52$ & 0.006 \\
\hline LDL (mg/dL) & $38 \pm 26$ & $-25 \pm 51$ & $10 \pm 31$ & $-40 \pm 42$ & $-42 \pm 48$ & $<0.001$ \\
\hline HDL (mg/dL) & $-2 \pm 14$ & $7 \pm 25$ & $3 \pm 12$ & $-0.3 \pm 10$ & $4 \pm 20$ & 0.809 \\
\hline Triglyceride (mg/dL) & $15 \pm 51$ & $-52 \pm 184$ & $-89 \pm 111$ & $-30 \pm 109$ & $-26 \pm 73$ & 0.36 \\
\hline $\mathrm{AST}(\mathrm{mg} / \mathrm{dL})$ & $-17 \pm 22$ & $-3 \pm 16$ & $-2 \pm 14$ & $-6 \pm 17$ & $-2 \pm 12$ & 0.469 \\
\hline $\operatorname{ALT}(\mathrm{mg} / \mathrm{dL})$ & $-20 \pm 54$ & $-10 \pm 28$ & $-3 \pm 15$ & $-8 \pm 32$ & $-9 \pm 27$ & 0.765 \\
\hline $\begin{array}{l}\text { Number of criteria for metabolic } \\
\text { syndrome }(0-5)\end{array}$ & $1.3 \pm 0.6$ & $1.5 \pm 1$ & $1.2 \pm 0.7$ & $1.3 \pm 1$ & $1 \pm 0.8$ & 0.455 \\
\hline
\end{tabular}




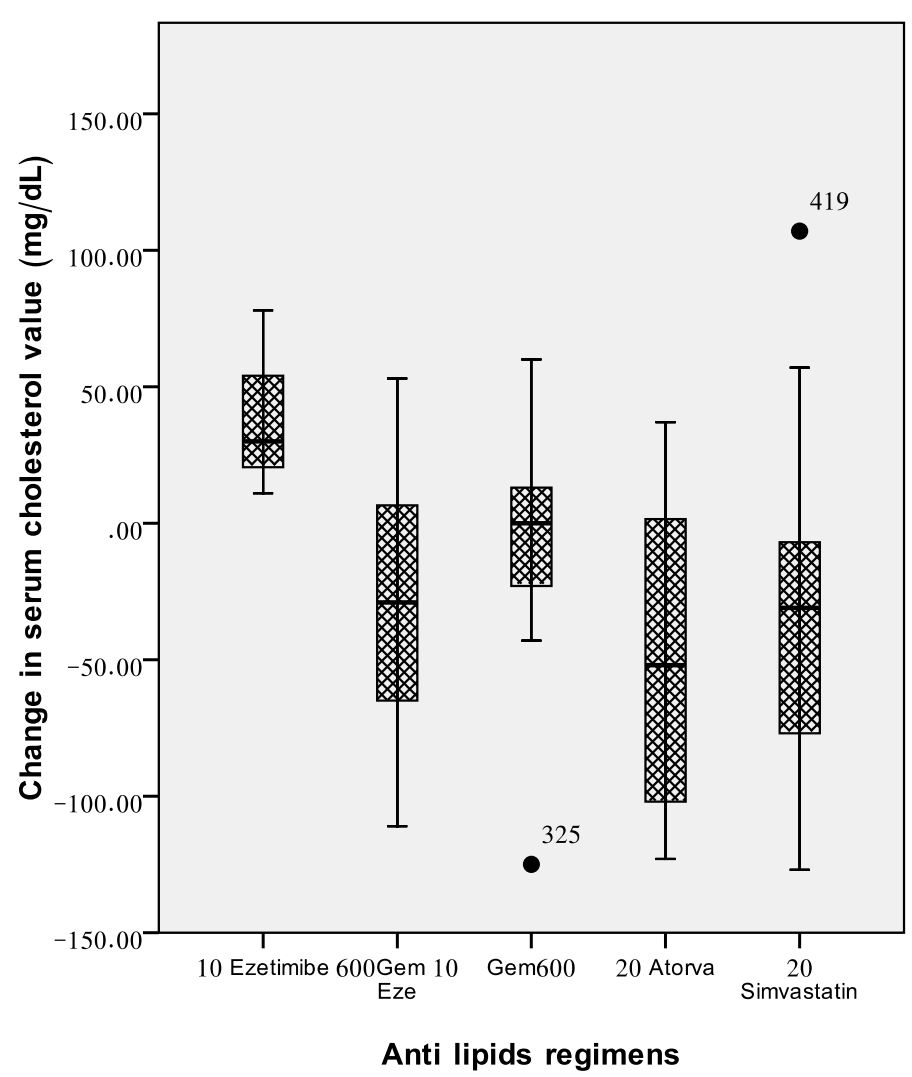

Figure 1. Changes in serum total cholesterol values after follow up

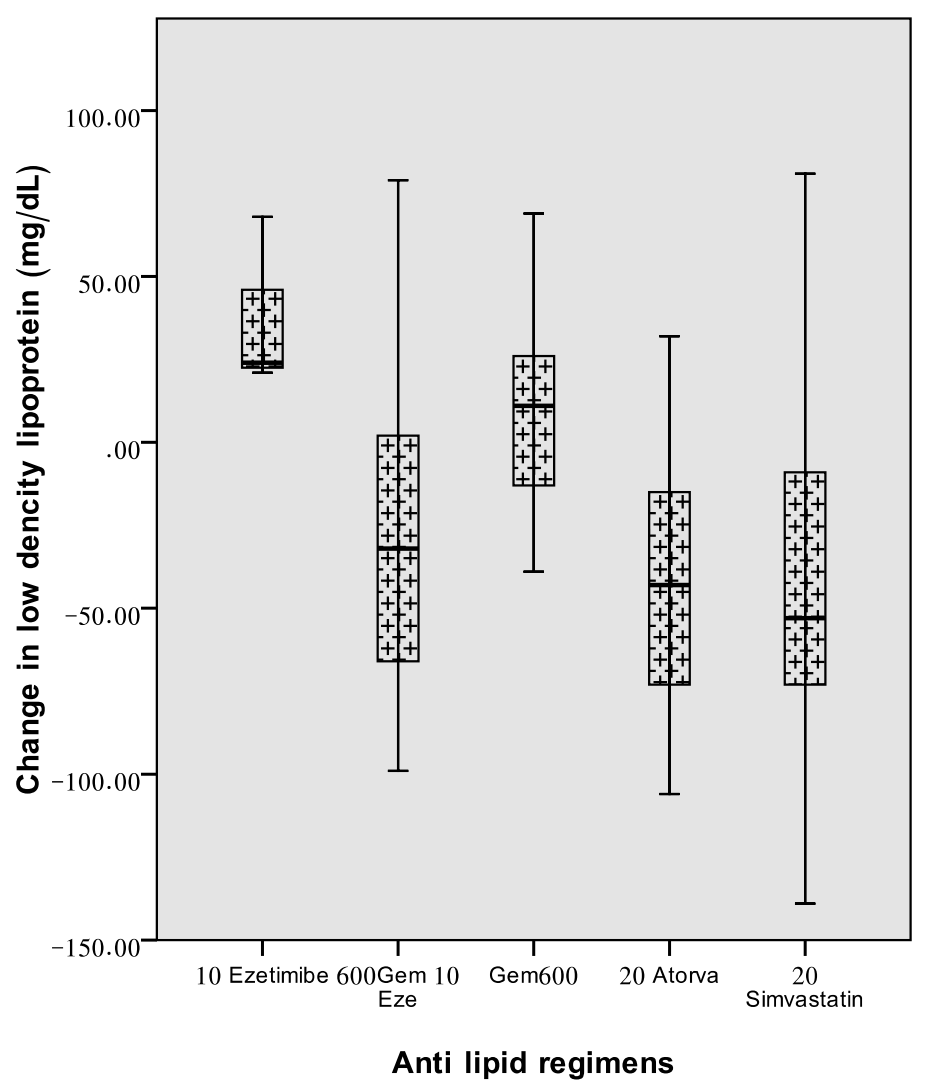

Figure 2. Changes in the serum LDL values after follow up 


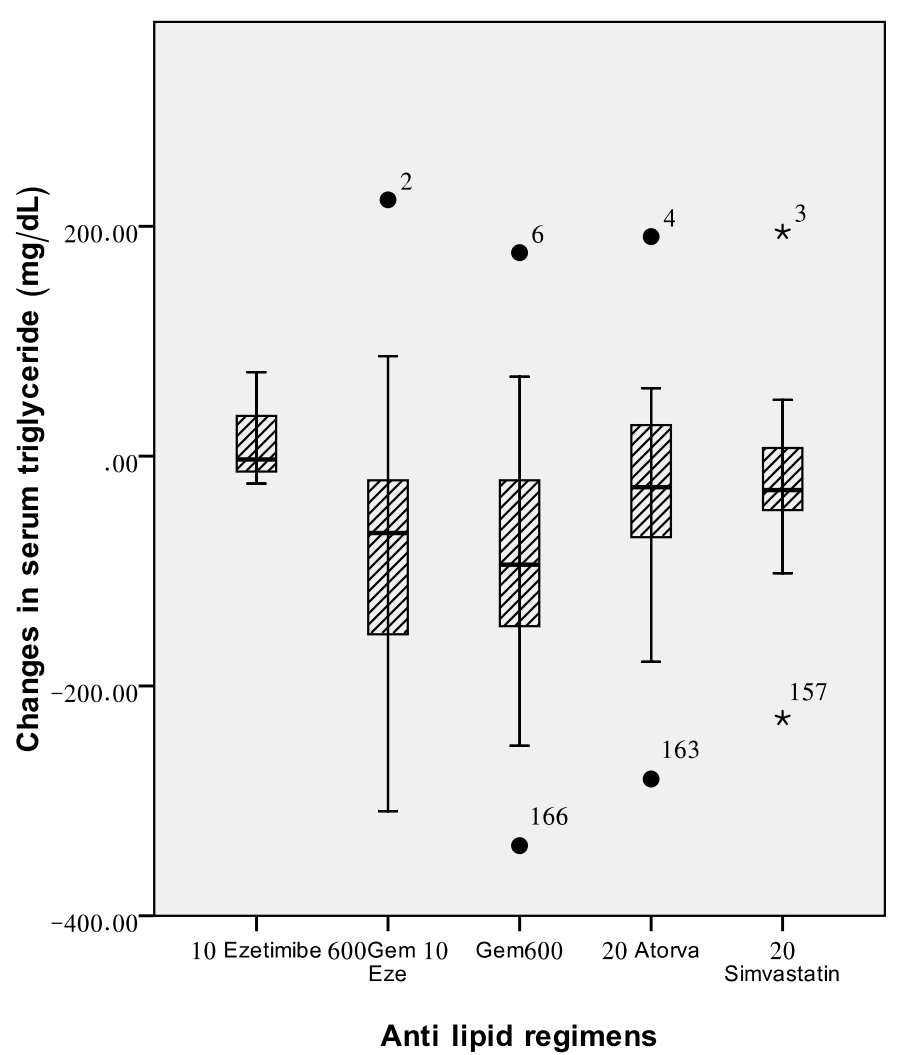

Figure 3. Changes in serum triglyceride values after follow up 